East African Medical Journal Vol. 87. No. 2 February 2010

ANTIBACTERIAL SUSCEPTIBILITY PATTERNS OF BLOOD STREAM ISOLATES IN PATIENTS INVESTIGATED AT THE AGA KHAN UNIVERSITY HOSPITAL, NAIROBI

R. Kohli, MBChB, Resident, G. Omuse, MBChB, Resident and G. Revathi, MBBS, MD (Clin. Micro.), Professor, Department of Microbiology, Aga Khan University Hospital, Nairobi, P.O. Box 30270-00100, Nairobi, Kenya

Request for reprints to: Prof. G. Revathi, Department of Microbiology, Aga Khan University Hospital, Nairobi, P.O. Box 30270-00100, Nairobi, Kenya

\title{
ANTIBACTERIAL SUSCEPTIBILITY PATTERNS OF BLOOD STREAM ISOLATES IN PATIENTS INVESTIGATED AT THE AGA KHAN UNIVERSITY HOSPITAL, NAIROBI
}

\author{
R. KOHLI, G. OMUSE and G. REVATHI
}

\begin{abstract}
Background: Microbial invasion of the blood stream can have serious immediate consequences and are a threat to every organ in the body. Timely detection and treatment is vital and necessitates hospital admission and immediate intervention.

Objectives: To investigate aetiology and anti-microbial resistance patterns of bacterial isolates in blood stream infections.

Design: A retrospective clinical-laboratory study carried over a five year period January 2003 to April 2008.

Setting: The Aga Khan University Hospital, Nairobi, Department of Pathology, Division of Microbiology.

Subjects: All blood culture specimens received from both in and out-patients' at the Aga Khan University Hospital's laboratory.

Results: Rates of oxacillin resistance for Staphylococcus aureus were $21 \%$. Streptococci were generally susceptible to beta-lactams. High-level gentamicin resistance was seen in $12 \%$ of Enterococci. Vancomycin resistance was conspicuously absent. Resistance rates of Pseudomonas aeruginosa to ciprofloxacin, gentamicin, amikacin, imipenem were between $11 \%$ and $23 \%$. Salmonella spp. showed multiple resistant patterns to co-trimoxazole, chloramphenicol and ampicillin with resistance rates of greater than $35 \%$. One hundred and twenty three patients $(\mathbf{1 1} \%)$ tested positive for HIV. Unlike in HIV negative individuals, Cryptococcus neoformans was an important isolate, positive in 5\%. A number of HIV positive patients had Staphylococcus aureus and coagulase negative staphylococcus isolates in their blood cultures. In such clinical circumstances it is difficult to determine the clinical significance of these isolates.

Conclusion: Antimicrobial susceptibility patterns revealed high level resistance among the gram positive organisms and also amongst extended spectrum beta lactamase (ESBL) producing E.coli and Klebsiella spp. This study highlights the challenges of deriving empiric drug regimens in the current clinical scenario. However, we do know it is important to cover adequately for gram positive organisms.
\end{abstract}

\section{INTRODUCTION}

Bloodstream infections are an important cause of morbidity and mortality among hospitalised patients and the surveillance of aetiological agents in these infections is essential for their prevention and treatment. Microbial invasion of the blood stream can have serious immediate consequences that is shock, multiple organ failure, disseminated intravascular coagulation (DIC) and death. Therefore timely detection and identification of blood borne pathogens is important (1).
Data on common organisms isolated from blood cultures from Kenya are limited. To our knowledge no similar studies looking at blood culture isolates have been done recently in Kenya. As there are no formal mechanisms of data collection at a national level. Our aim was to identify the common blood stream isolates at the Aga Khan University Hospital, Nairobi, Kenya. This type of study might contribute to formulation of empirical regimens to treat suspected blood stream infections in emergency situations. 
Study setting: The study was undertaken at the Aga Khan University Hospital, Nairobi, Kenya, a 250 bed postgraduate teaching hospital. In addition to high technology diagnostic facilities, the hospital offers general medical services as well as all specialist clinics.

Study design: This was a retrospective clinicallaboratory study carried out over five years between January 2003 and April 2008. One thousand and ninety two patient isolates were analysed during the study duration.

\section{MATERIALS AND METHODS}

Laboratory methods: Laboratory registers were reviewed to identify all positive blood culture isolates over the study period. Subsequently, patient files were reviewed in the medical records department. A data collection tool was designed to extract the patient information systematically and recorded onto Excel sheets to be analysed.

Blood culture techniques / Antibioticsusceptibility testing: Blood specimens were cultured in BACTEC 9120 and BACTEC 9050 (Becton-Dickinson, USA) automated systems and bacterial identification was done with standard bacteriological techniques, like colony morphology, gram smear techniques and rapid bench tests e.g. catalase test, oxidase tests etc. Biochemical tests were done using bioMerieux products (API 20E, API 20NE, API Coryne, API Candida etc) (2). Serological testing was done using Remel diagnostic products. Antimicrobial susceptibility testing was done by disc diffusion method using the modified Kirby-Bauer technique and performed according to Clinical and Laboratory Standards Institute (CLSI), January, 2008. Strict internal quality and external quality controls are in place in the microbiology laboratory. External quality control is done through the UK NEQAS programme.

It is customary practice in the microbiology department to call the clinician requesting the blood culture as soon as the blood culture flags positive. A gram stain is performed and a preliminary report submitted. If pneumococcus is suspected then a direct sensitivity test is set up. This is important because the clinician can then decide on which antibiotic to use in the interim before final culture results are released.

Statistical analysis: Statistical analysis was done using SPSS software (SPSS Inc., Chicago, IL. version 15.0). Data were presented as pie charts and tables. Frequencies were calculated for categorical variables.

\section{RESULTS}

The total number of blood cultures done during the study period were 18,750 . The total number of positive blood cultures obtained was 1,092. Organisms isolated were either gram positive bacteria, gram negative bacteria or yeasts. Eleven patients had polymicrobial isolates. Despite implementation of strict phlebotomy and skin disinfection with chlorhexidine, $3 \%$ of the cultures were still contaminated with aerobic spore bearer bacilli.

The number of males was $603(55.2 \%)$ and the number of females was $489(44.8 \%)$. The number of blood culture specimens from in-patients was 1015 and those obtained from out-patients was 77. Of the in-patients, most blood culture isolates were from patients admitted to the medical wards: Intensive Care Unit - 51 (5\%); High Dependency Unit - 50 (5\%); Surgical Ward - 141 (14\%); Medical Ward - 517 (51\%); Private Wing - 169 (17\%). The majority of the patients were medical; New Born Unit - 87 (9\%).

Out of the 1092 isolates: Gram positive bacteria - 695 (64\%); Gram negative bacteria - $359(33 \%)$ and Yeasts $-38(3 \%)$.

The commonly isolated gram positive organisms were Staphylococcus aureus with $368(54 \%)$ isolates and coagulasenegative Staphylococcus with $133(20 \%)$ isolates. Other commonly isolated gram positive organisms have been shown in Figure 1.

Figure 1

Commonly isolated gram positive organisms

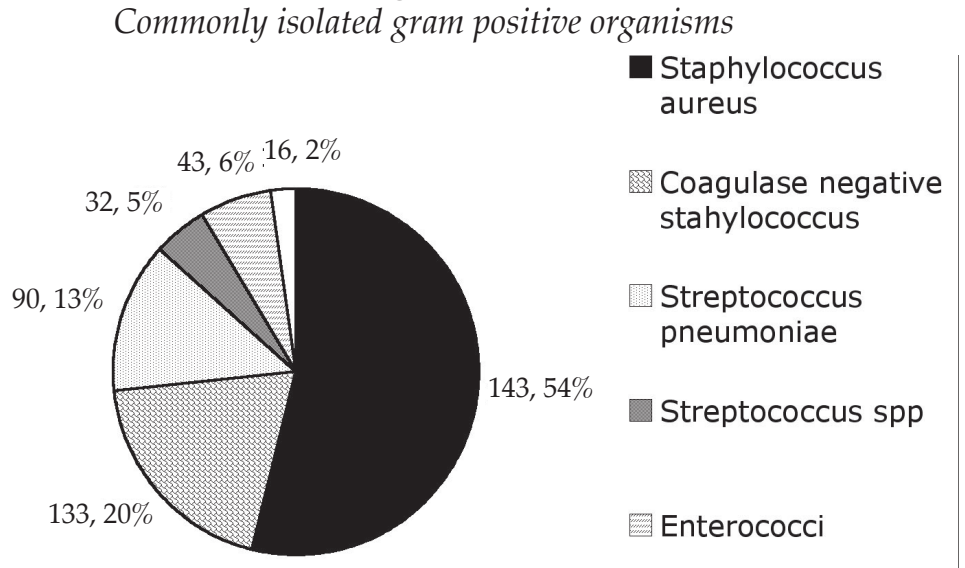


Figure 2 shows most commonly isolated gram negative organisms with the most common isolate being Salmonella spp with 143 isolates (54\%). Escherichia coli was also a common isolate with 69 $(26 \%)$ isolates.

Table 1 shows clinical correlation with organism type. In 434 patients with a gram positive isolate there was no clinical information provided. This was similar to gram negative isolates in which 249 had no clinical information. In patients in whom there was clinical information, pyrexia of unknown origin was an indication for 63 patients with gram positive isolates and 58 with gram negative isolates. Table 1 shows other clinical correlates and the organism type.

One hundred and twenty three patients tested positive for HIV, 421 were negative, 548 patients were notscreened. The mostcommon blood culture isolate in HIV positive patients was Staphylococcus spp with(42\%) 52 isolates. This was similar in HIV negative patients with 195 (46\%) Staphylococcus spp isolates. Cryptococcus neoformans was also a common isolate in HIV positive patients with six isolates $(5 \%)$, other common blood culture isolates have been shown in Table 2 .

Figure 2

Commonly isolated gram negative bacteria

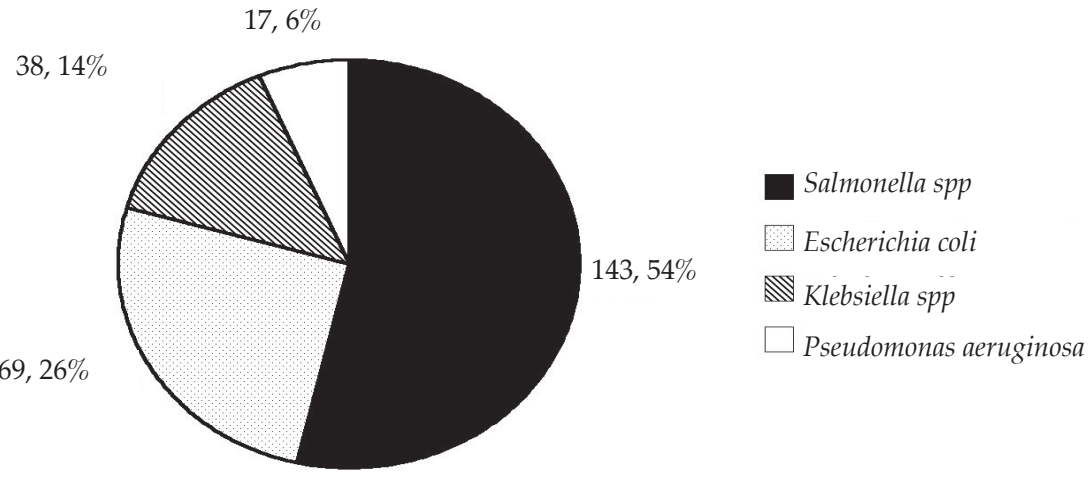

Commonly isolated yeasts included the following: Candida spp - 20 (53\%); Cryptococcus neoformans - 18 (47\%).

Table 1

Clinical Correlation with organism type

\begin{tabular}{lcc} 
Clinical Indication For Blood Culture & Gram positive & Gram negative \\
\hline Pyrexia of unknown origin (121) & 63 & 58 \\
Septicaemia (33) & 20 & 13 \\
Neonatal sepsis (19) & 14 & 5 \\
Pneumonia (44) & 35 & 9 \\
Gastroenteritis (22) & 11 & 11 \\
Neonatal jaundice (8) & 6 & 2 \\
Meningitis (5) & 3 & 2 \\
Hypertension (3) & 3 & \\
HIV (33) & 18 & 15 \\
Diabetes (12) & 8 & 4 \\
End stage renal disease (5) & 4 & 1 \\
Infection secondary to polytrauma (104) & 72 & 32 \\
No clinical information provided (683) & 434 & 249 \\
\end{tabular}


Table 2

Most common blood culture isolates in HIV positive patients compared with HIV negative patients

\begin{tabular}{|c|c|c|c|c|}
\hline \multicolumn{2}{|c|}{ HIV Positive $(\mathrm{n}=123)$} & \multicolumn{3}{|c|}{ HIV Negative $(\mathrm{n}=421)$} \\
\hline Organism & No. $(\%)$ & Organism & No. & $(\%)$ \\
\hline Staphylococcus spp & 5242 & Staphylococcus spp & 195 & 46 \\
\hline Streptococcus spp & 20 & Salmonella spp & 56 & 13 \\
\hline Salmonella spp & 19 & Streptococcus spp & 48 & 11 \\
\hline Cryptococcus neoformans & 6 & Escherichia coli & 27 & 6 \\
\hline Escherichia coli & 5 & Klebsiella & 15 & 4 \\
\hline Others & 22 & Others & 80 & 19 \\
\hline
\end{tabular}

Table 3

Antimicrobial resistance patterns to gram positive organisms

\begin{tabular}{|c|c|c|c|c|c|c|c|c|c|}
\hline & \multicolumn{2}{|c|}{$\begin{array}{l}\text { Staph. } \\
\text { aureus } \\
(n=364)\end{array}$} & \multicolumn{2}{|c|}{$\begin{array}{l}\text { Coagulase } \\
\text { Negative } \\
\text { Staph. } n=137 \text { ) }\end{array}$} & \multicolumn{2}{|c|}{$\begin{array}{l}\text { Strep. } \\
\text { Pneumo } \\
(\mathrm{n}=90)\end{array}$} & $\begin{array}{l}\text { Strep. spp } \\
(\mathrm{n}=32)\end{array}$ & $\begin{array}{l}\text { Entero } \\
(n=43)\end{array}$ & $\begin{array}{l}\text { Coryneb } \\
(\mathrm{n}=16)\end{array}$ \\
\hline & No. & $(\%)$ & No. & $(\%)$ & No. & $(\%)$ & No. $(\%)$ & No. $(\%)$ & No. $(\%)$ \\
\hline Ampicillin & 162 & 45 & 64 & 47 & 22 & 24 & $\begin{array}{ll}4 & 14\end{array}$ & $\begin{array}{ll}6 & 14\end{array}$ & 638 \\
\hline $\begin{array}{l}\text { Amoxicillin- } \\
\text { clavuloinic acid }\end{array}$ & 76 & 21 & 37 & 27 & 5 & 6 & 13 & 4 & 16 \\
\hline Gentamicin & 3 & 0.8 & & 3 & ND & & ND & $5 \quad 12$ & ND \\
\hline Streptomycin & ND & & ND & & ND & & ND & $5 \quad 12$ & ND \\
\hline Ciprofloxacin & 67 & 18 & 45 & 33 & 6 & 7 & 413 & $7 \quad 16$ & 213 \\
\hline Vancomycin & 2 & 0.5 & 1 & 0.7 & 0 & & ND & 12 & 0 \\
\hline Doxycycline & 110 & 30 & 49 & 36 & 7 & 8 & $4 \quad 14$ & ND & 319 \\
\hline Azithromycin & 126 & 35 & 65 & 47 & 6 & 7 & $6 \quad 19$ & ND & 213 \\
\hline Cefuroxime & 74 & 20 & 27 & 20 & 8 & 9 & 13 & ND & 0 \\
\hline Chloramphenicol & 16 & 4 & 16 & 12 & 3 & 3 & 0 & ND & 16 \\
\hline Oxacillin & 76 & (21) & 27 & 20 & 22 & 24 & ND & ND & ND \\
\hline Netilmycin & 7 & (2) & 7 & 5 & ND & & ND & ND & 0 \\
\hline Erythromycin & 126 & (35) & 65 & 47 & 6 & 7 & $6 \quad 19$ & ND & 213 \\
\hline Co-trimoxazole & 68 & (19) & 23 & 17 & D 0 & 11 & $4 \quad 14$ & ND & ND \\
\hline
\end{tabular}

Coryneb $=$ Corynebacterium, Entero $=$ Enterococcus, $N D=$ Not Done

Ampicillin resistance was high in both Staphylococcus aureus and coagulase negative Staphylococcus with $45 \%$ and $47 \%$ of the isolates being resistant respectively. Staphylococcus aureus exhibited 30\% and $35 \%$ resistance to azithromycin and doxycline respectively. Vancomycin showed low level resistance to Staphylococcus aureus with $0.5 \%$ resistance. Table 3 depicts further the antibiotic resistance profiles of gram positive organisms.
Salmonella spp showed increased resistance to ampicillin (31\%) and co-trimoxazole (44\%). E. colialso showed increased resistance to co-trimoxazole $(71 \%)$ and amoxicillin-clavulanic acid (38\%). Pseudomonas generally showed low level resistance to various antibiotics tested and the highest resistance was shown with gentamicin. $31 \%$ resistance. Table 4 shows antibiotic resistance profiles to gram negative organisms. 
Table 4

Antimicrobial resistance patterns to gram negative organisms

\begin{tabular}{|c|c|c|c|c|c|c|c|c|c|}
\hline & \multicolumn{2}{|c|}{$\begin{array}{c}\text { Pseudo \& NFs } \\
(\mathrm{n}=48)\end{array}$} & \multicolumn{2}{|c|}{$\begin{array}{l}\text { E.coli } \\
(n=69)\end{array}$} & $\begin{array}{c}\text { Klebs } \\
(\mathrm{n}=38) \\
\text { No. }(\%)\end{array}$ & $\begin{array}{l}\text { Proteus } \\
(\mathrm{n}=4) \\
\text { No. }(\%)\end{array}$ & $\begin{array}{c}\text { Entero } \\
(\mathrm{n}=18) \\
\text { No. }(\%)\end{array}$ & $\begin{array}{c}\text { Kluy } \\
(\mathrm{n}=23) \\
\text { No. }(\%)\end{array}$ & $\begin{array}{c}\text { Salm } \\
(\mathrm{n}=143) \\
\text { No. }(\%) \\
\end{array}$ \\
\hline Ampicillin & & $\mathrm{ND}$ & & $\mathrm{D}$ & ND & ND & ND & ND & $44 \quad(31)$ \\
\hline Co-trimoxazole & & ND & 49 & (71) & $14 \quad 37$ & 250 & 317 & $10 \quad 43$ & $63 \quad(44)$ \\
\hline $\begin{array}{l}\text { Amoxicillin- } \\
\text { clavulanic acid }\end{array}$ & & ND & 26 & (38) & $13 \quad 34$ & 0 & 739 & 35 & ND \\
\hline Ciprofloxacin & & 19 & & (34) & $13 \quad 34$ & 125 & 422 & 30 & $14 \quad 10$ \\
\hline Doxycycline & & ND & 41 & (59) & $16 \quad 42$ & 375 & 422 & 11 & ND \\
\hline CAF & & ND & & (19) & $10 \quad 26$ & 125 & 422 & 22 & $57 \quad 40$ \\
\hline Gentamicin & & 31 & 20 & (29) & $8 \quad 21$ & ND & 211 & 22 & ND \\
\hline Cefuroxime & & ND & 15 & (22) & $14 \quad 37$ & 250 & 317 & $6 \quad 26$ & ND \\
\hline Cefotaxime & & ND & 10 & (14) & $4 \quad 11$ & 125 & 15 & 0 & ND \\
\hline Ceftriaxone & & 14 & 10 & (14) & $5 \quad 13$ & 0 & 0 & 0 & 0 \\
\hline $\begin{array}{l}\text { Nalidixic } \\
\text { acid }\end{array}$ & & $\mathrm{ND}$ & $\mathrm{N}$ & $\mathrm{D}$ & ND & ND & $\mathrm{ND}$ & ND & $14 \quad 10$ \\
\hline Amikacin & 8 & 17 & 9 & (13) & 8 & ND & 15 & 26 & ND \\
\hline Cefepime & 2 & 4 & 7 & (10) & $10 \quad 26$ & ND & 211 & $7 \quad 30$ & ND \\
\hline Ceftazidime & 4 & 8 & 10 & (14) & $5 \quad 13$ & 0 & 0 & 0 & 0 \\
\hline Imipenem & 3 & 6 & 1 & (1) & 25 & ND & 15 & $4 \quad 17$ & ND \\
\hline Tazo/Pip & 1 & 2 & 11 & (16) & $5 \quad 13$ & ND & 15 & 17 & ND \\
\hline Piperacillin & 5 & 10 & 10 & (14) & $10 \quad 26$ & 125 & ND & 30 & ND \\
\hline
\end{tabular}

Chlor = chloramphenicol, tazo/pip = tazobactam/Piperacillin, Pseudo and NFs = Pseudomonas and Non Fermenters, Klebs $=$ Klebsiella, Entero $=$ Enterobacter, Kluy $=$ Kluyvera, Salm $=$ Salmonella, ND $=$ Not Done

\section{DISCUSSION}

In this study, the large number of blood cultures done ensured a large sample to analyse. This is in sharp contrast to publichospitals within the country, where very small number of blood cultures are performed due to scarcity of resources although they manage a much larger number of patients, for example, one of the National Referral Hospitals in Kenya has a bed capacity of more than 2000.

Out of a total of 18,750 blood cultures done, only $1,092(5.8 \%)$ were positive. This suggests that either patients had already received antibiotics prior to sampling, meaning fewer positive isolates, or that clinicians were investigating with minimal clinical evaluation. This is highlighted by our study which shows that in $683(63 \%)$ of the patients in whom a blood culture was done, no clinical information was provided.

Out of the positive cultures only $77(7 \%)$ were done on an outpatient basis. This may insinuate that clinicians readily admit patients when there is an indication for blood culture.

Of the gram positive organisms the two important pathogens were Staphylococcus spp
(S. aureus. 54\%; Coagulase Negative Staphylococcus [CoNS] 20\%) and Streptococcus pneumoniae (13\%). This finding was similar to other studies which also looked at blood culture isolates $(3,4)$. Staphylococcus spp. especially CoNS are the most common isolates as they are commonly found on the skin surface. Since most patients had only one out of three cultures taken a positive finding of CoNS as an isolated finding may cast doubt on the significance of this isolate.

The predominant gram negative organisms were Salmonella (54\%) and Escherichia coli (26\%). Of the Salmonella spp, 85 (59\%) were Salmonella typhi. The high prevalence of Salmonella isolated may be attributed to the endemicity of this organism in this region. Fewer Salmonella typhi cases are reported in developed countries. A study done in Iran also showed high rates of Salmonella in their blood culture isolates (5).

Enterobacter spp. was isolated in only a minority of blood culture specimens $(4 \%)$; this differs from the West where Enterobacter spp. is an important pathogen. This finding highlights the disparity between the two populations and emphasizes the danger of adopting Western data sets. 
Eleven (1\%) patientshad polymicrobial isolates. Most of these isolates were from in-patients. A similar study conducted in India also showed that $4.5 \%$ of isolates were polymicrobial and these were also from in-patients (3).

The primary indication for a blood culture was pyrexia of unknown origin followed by infection secondary to polytrauma. In patients with polytrauma, the predominant isolate was gram positive cocci $(57 \%)$ followed by gram negative bacilli ( $38 \%$ ). This finding may aid in formulating empiric antibiotic treatment in emergency situations in preventing infection in polytrauma patients.

A total of $123(11 \%)$ patients tested positive for HIV. The common isolates in these patients were Staphylococcus aureus (42\%), Streptococcus spp including pneumococcus $(16 \%)$ and Salmonella spp $(15 \%)$. A prospective study done in Kenya showed that common bloodstream isolates in patients with HIV were predominantly Salmonella spp. and Streptococcus pneumoniae (6). In this study, most of the patients were from a resource poor setting unlike in our study setting, which may account for the higher number of Salmonella cases seen. The high isolation of Streptococcus spp in HIV positive patients is because HIV related immuno-suppression increases the risk of invasive pneumococcal disease (7).

Rates of oxacillin resistance of Staphylococcus aureus in our study were $21 \%$, and for CoNS, $20 \%$. This was higher in comparison to a study done in Abidjan, which showed an MRSA rate of $14.2 \%$ (18). This was significantly lower compared to studies done in Taiwan(9), where it was $75 \%$. Surveillance studies done in Europe showed variation in MRSA rates from country to country, $0.7 \%$ in Sweden and up to $47.5 \%$ in UK (10).

Streptococci were generally susceptible to all beta-lactams. Surveillance done in UK and Ireland showed similar findings (11). High-level gentamicin resistance was seen in $12 \%$ of Enterococci. This differed from the UK/Ireland where resistance was as high as $43 \%$ (11).

Vancomycin resistanceto Enterococci was rare. This varies from the West where resistance to vancomycin is significant. In the USA colonisation with vancomycin resistantEnterococci (VRE) is endemicin many hospitals and increasingly causes infections (12). In France and Spain, vancomycin resistantEnterococci prevalence was $<1 \%$ and in UK and Ireland it is $>14 \%$ (10). Vancomycin use has been much higher in the USA compared to that in Kenya probably explaining this difference in resistance patterns.

Escherichia coli isolates were commonly resistant to co-trimoxazole $(71 \%)$ and doxycycline (59\%). A number of E.coli and Klebsiella spp. were extended spectrum beta-lactamase (ESBL) producers, $14 \%$ and $13 \%$ respectively. A study done in Finland on nosocomial infections showed ESBL-producing $E$. coli and Klebsiella spp. bacteremia to be low (5\% and $0 \%$ of isolates respectively) (13). Resistance rates of Pseudomonas aeruginosa to imipenem were 6\%. In Europe, resistance to imipenem for Pseudomonas aeruginosa was $<3 \%$ in Sweden/UK and 32.2\% in Turkey (10).

In conclusion, this study shows that it is difficult for us to come up with an empiric regimen for blood stream infections due to the presence of emerging resistance to commonly used drugs among different organisms. Also, with the impact of HIV, the aetiology of blood stream isolates differs from the general population. However, the study has shown that specific empiric regimens in certain clinical situations e.g. polytrauma can be formulated. Most of the patients were successfully treated with broad spectrum antibiotics. Mortality rate was low at $0.6 \%$.

The limitations of the study were that since this was not a designed prospective study, there was no control on the sampling of subjects as the requests are raised at the clinicians' discretion and may result in either over investigation or under-investigation of the patient. Most of the requests had no accompanying clinical information so the indication of blood culture was unknown. The patient files also did not capture all the clinical information.

However, in the absence of large prospective studies, review of clinical laboratory data gives us insights into trends of the bacterial isolates and antibiotic susceptibility patterns by providing a random epidemiologically acceptable sample. The study required no additional resources which was an advantage.

We recommend that a well designed prospective multi-centre study needs to be conducted to achieve reliable and clinically dependable results. In addition to institution-based studies, large community studies are also essential to give a realistic picture. Prospective studies will positively overcome the lack of comprehensive clinical data that we encountered during this study.

\section{ACKNOWLEDGEMENTS}

To all the technologists at the Aga Khan Laboratory's Microbiology Division. 


\section{REFERENCES}

1. Forbes, B.A. Bailey, and Scotts Diagnostic Microbiology. Eleventh ed. 2002.

2. Murray, P. Manual of Clinical Microbiology. Eighth ed. 2003.

3. Atul Garg, S.A., Jaya G., Goyal, R.K. and Sen, M. R. Bacteriological profile and antimicrobial resistance of blood culture isolates from a University Hospital. J. Ind. Acad. Clin. Med. 2007; 8: 139 - 143.

4. Rahbar, M. RG-A. and Hashemi, S. Nosocomialblood stream infections in Imam Khomeini Hospital, Urmia, Islamic Republic of Iran, 1999-2001. Eastern Mediter. Health J. 2005; 11: 478 - 484.

5. Sobhani, A. H.S., and Javanbakht, S. Drug resistance pattern in isolated bacteria from blood cultures. Acta Med. Iranica. 2004; 42: 46 - 49.

6. Arthur, G., Nduba, V.N., Kariuki, S.M., et al. Trends in bloodstream infections among human immunodeficiency virus-infected adults admitted to a hospital in Nairobi, Kenya, during the last decade. Clin. Infect. Dis. 2001; 33: 248-256.

7. Gilks, C., Ojoo, S.A. and Ojoo, J.C. Invasive pneumococcal disease in a cohort of predominantly HIV - 1 infected female sex workers in Nairobi, Kenya. Lancet. 1996; 347: 718-723.

8. Akoua-Koffi, C., Guessennd, N., Gbonon, V., et al. Methicillin resistance of Staphylococcus aureus in
Abidjan (1998-2001): a new hospital problem. Med Mal. Infect. 2004; 34: 132-136.

9. Wu, C. J. L.H., Lee, N.Y., Shih, H.I. et al. Predominance of gram-negative bacilli and increasing antimicrobial resistance in nosocomial bloodstream infections at a university hospital in southern Taiwan, 1996-2003. J. Microbiol. Immuno. Infect. 2006; 39: 135-143.

10. Sader, H.F.T., Stilwell, M. and Jones, R., editor. 10 years of surveillance of blood stream infections in European medical centres by the SENTRY Antimicrobial Surveillance Program (1997-2006). 17 $7^{\text {th }}$ European Congress of Clinical Microbiology and Infectious Diseases; 2007, Munich, Germany.

11. Reynolds, R., Potz, N., Colman, M., et al. Antimicrobial susceptibility of the pathogens of bacteraemia in the UK and Ireland 2001 - 2002: the BSAC Bacteraemia Resistance Surveillance Programme. J. Antimicro. Chemo. 2004; 53:

12. Marc, J.M. Bonten, R.W. and Robert, A.W. Vancomycin resistant enterococci: why are they here and where do they come from? The Lancet Infect. Dis. 2001; 1: 314-325.

13. Lyytikainen, O., Kanerva, M., Agthe, N., Mottonen, T. and Ruutu, P. Healthcare-associated infections in Finnish acute care hospitals: a national prevalence survey, 2005. J. Hosp. Infect. 2008; 69: 288-294. 